\title{
Assessing the Role of Projections from the Ventral Hippocampus (vHIP) to the Medial Prefrontal Cortex (mPFC) in the Neural Circuitry of Social Familiarity-induced Anxiolysis (SoFiA) using Gi-DREADDs
}

\author{
M. Regina Barron ${ }^{3}$ and William Truitt ${ }^{1,2,3}$ \\ ${ }^{1}$ Department of Anatomy and Cell Biology; ${ }^{2}$ Stark Neurosciences Research Institute; ${ }^{3}$ Indiana \\ University School of Medicine, Indianapolis, IN
}

The social interaction habituation (SI-hab) protocol has been used to demonstrate that rats acquire Social Familiarity-induced Anxiolysis (SoFiA), a form of safety learning where rats display anxiolysis in the presence of a familiar conspecific. SoFiA acquisition and expression can be simplified into four different constructs: social memory, anxiety, safety learning and anxiolysis. The neural circuitry of SoFiA; however, has not been fully identified. As a step towards elucidating the SoFiA neural circuitry we are identifying the role of projections from the ventral hippocampus (vHIP) to the medial prefrontal cortex (mPFC) in SoFiA acquisition.

Prior work from our lab has shown that the mPFC is needed for acquisition and expression of SoFiA. The mPFC may be the location of convergence of anxiety and social memory processing, making it a loci for safety learning. The goal of the current work is to identify the inputs to the mPFC that are pivotal for SoFiA acquisition. vHIP has been implicated in social memory and projects to the mPFC leading to the hypothesis that SoFiA acquisition requires vHIP projections to mPFC.

This hypothesis will be tested via intersectional genetics where neural tracts are selectively targeted through viruses. One virus is introduced at the soma of the neuron, and another is introduced at the end of the axon. The first virus, adeno-associated virus 8 (AAV8) will contain Cre-dependent code for an inhibitory DREADD (designer receptors exclusively activated by designer drugs.) The second virus, canine adenovirus 2 (CAV2) is picked up by axon terminals and transported to the soma where it will express Cre. Unfortunately, the uptake and transport of these viruses require specific receptors and the vHIP-mPFC pathway has poor CAV2 uptake. CAV2 levels will be increased by the introduction of Coxsackievirus and Adenovirus receptor (CAR) via AAV8 two weeks prior to CAV2-Cre injection. 